\title{
Extension of B-spline Material Point Method for unstructured triangular grids using Powell-Sabin splines
}

\author{
Pascal de Koster ${ }^{1} \cdot$ Roel Tielen ${ }^{1}$. Elizaveta Wobbes ${ }^{1} \cdot$ Matthias Möller $^{1}$
}

Received: 16 July 2019 / Revised: 7 February 2020 / Accepted: 6 March 2020 / Published online: 26 March 2020

(c) The Author(s) 2020

\begin{abstract}
The Material Point Method (MPM) is a numerical technique that combines a fixed Eulerian background grid and Lagrangian point masses to simulate materials which undergo large deformations. Within the original MPM, discontinuous gradients of the piecewise-linear basis functions lead to the so-called grid-crossing errors when particles cross element boundaries. Previous research has shown that B-spline MPM (BSMPM) is a viable alternative not only to MPM, but also to more advanced versions of the method that are designed to reduce the grid-crossing errors. In contrast to many other MPM-related methods, BSMPM has been used exclusively on structured rectangular domains, considerably limiting its range of applicability. In this paper, we present an extension of BSMPM to unstructured triangulations. The proposed approach combines MPM with $C^{1}$-continuous high-order Powell-Sabin spline basis functions. Numerical results demonstrate the potential of these basis functions within MPM in terms of grid-crossing-error elimination and higher-order convergence.
\end{abstract}

Keywords Material Point Method · B-splines · Powell-Sabin splines · Unstructured grids · Grid-crossing error

\section{Introduction}

The Material Point Method (MPM) has proven to be successful in solving complex engineering problems that involve large deformations, multi-phase interactions and historydependent material behaviour. Over the years, MPM has been applied to a wide range of applications, including modelling of failure phenomena in single- and multi-phase media $[1,35,40]$, crack growth $[14,20]$ and snow and ice dynamics $[12,31,34]$. Within MPM, a continuum is discretised by defining a set of Lagrangian particles, called material points, which store all relevant material properties. An Eulerian background grid is adopted on which the equations of motion are solved in every time step. The solution on the background grid is then used to update all material point properties such as displacement, velocity and stress. In this way, MPM incorporates both Eulerian and Lagrangian descriptions. Similarly to other combined Eulerian-Lagrangian techniques, MPM attempts to avoid the numerical difficulties arising from non-

\section{Pascal de Koster}

p.b.j.dekoster@tudelft.nl

1 Department of Applied Mathematics, Section of Numerical Analysis, Delft University of Technology, Van Mourik Broekmanweg 6, 2628 XE Delft, The Netherlands linear convective terms associated with an Eulerian problem formulation, while at the same time preventing grid distortion, typically encountered within mesh-based Lagrangian formulations (e.g. [10,32]).

Classically, MPM uses piecewise-linear Lagrange basis functions, also known as 'tent' functions. However, the gradients of these basis functions are discontinuous at element boundaries. This leads to the so-called grid-crossing errors [2] when material points cross this discontinuity. Gridcrossing errors can significantly influence the quality of the numerical solution and may eventually lead to a lack of convergence (e.g. [28]). Different methods have been developed to mitigate the effect of grid-crossings. For example, the Generalised Interpolation Material Point (GIMP) [2] and Convected Particle Domain Interpolation (CPDI) [25] methods eliminate grid-crossing inaccuracies by introducing an alternative particle representation. The GIMP method represents material points by particle-characteristic functions and reduces to standard MPM, when the Dirac delta function centred at the material point position is selected as the characteristic function. For multivariate cases, a number of versions of the GIMP method are available such as the uniform GIMP (uGIMP) and contiguous-particle GIMP (cpGIMP) methods. The CPDI method extends GIMP in order to accurately capture shear distortion. Much research has been performed 
to further improve the accuracy of the CPDI approach and increase its range of applicability $[16,22,26]$. On the other hand, the Dual Domain Material Point (DDMP) method [41] preserves the original point-mass representation of the material points, but adjusts the gradients of the basis functions to avoid grid-crossing errors. The DDMP method replaces the gradients of the piecewise-linear Lagrange basis functions in standard MPM by smoother ones. The DDMP method with sub-points [7] proposes an alternative manner for numerical integration within the DDMP algorithm.

The B-spline Material Point Method (BSMPM) [28, 29] solves the problem of grid-crossing errors completely by replacing piecewise-linear Lagrange basis functions with higher-order B-spline basis functions. B-spline and piecewise-linear Lagrange basis functions possess many common properties. For instance, they both satisfy the partition of unity, are non-negative and have a compact support. The main advantage of higher-order B-spline basis functions over piecewise-linear Lagrange basis function is, however, that they have at least $C^{0}$-continuous gradients which preclude grid-crossing errors from the outset. Moreover, spline basis functions are known to provide higher accuracy per degree of freedom as compared to $C^{0}$-finite elements [15]. On structured rectangular grids, adopting B-spline basis functions within MPM not only eliminates grid-crossing errors but also yields higher-order spatial convergence $[3,11,28,29$, 37]. Previous research also demonstrates that BSMPM is a viable alternative to the GIMP, CPDI and DDMP methods $[11,19,30,39]$. While the CPDI and DDMP methods can be used on unstructured grids $[16,22,41]$, to the best of our knowledge, BSMPM for unstructured grids does not yet exist. This implies that its applicability to real-world problems is limited compared to the CPDI and DDMP methods.

In this paper, we propose an extension of BSMPM to unstructured triangulations to combine the benefits of Bsplines with the geometric flexibility of triangular grids. The proposed method employs quadratic Powell-Sabin (PS) splines [23]. These splines are piecewise higher-order polynomials defined on a particular refinement of any given triangulation and are typically used in computer-aided geometric design and approximation theory [9,18,24,27]. PS-splines are $C^{1}$-continuous and hence overcome the grid-crossing issue within MPM by design. We would like to remark that although this paper focuses on PS-splines, other options such as refinable $C^{1}$ splines [21] can be used to extend MPM to unstructured triangular grids. The proposed PS-MPM approach is analysed based on several benchmark problems.

The paper is organised as follows. In Sect. 2, the governing equations are provided together with the MPM solution strategy. In Sect. 3, the construction of PS-spline basis functions and their application within MPM is described. In Sect. 4, the obtained numerical results are presented. In Sect. 5, conclusions and recommendation are given.

\section{Material Point Method}

We will summarise the MPM as introduced by Sulsky et al. [32] to keep this work self-contained. First, the governing equations are presented, afterwards the MPM strategy to solve these equations is introduced.

\subsection{Governing equations}

The deformation of a continuum is modelled using the conservation of linear momentum and a material model. It should be noted that MPM can be implemented with a variety of material models that either use the rate of deformation (i.e. the symmetric part of the velocity gradient) or the deformation gradient. However, for this study it is sufficient to consider the simple linear-elastic and neo-Hookean models that are based on the deformation gradient. Using the Einstein summation convention, the system of equations in a Lagrangian frame of reference for each direction $x_{k}$ is given by

$\frac{\partial u_{k}}{\partial t}=v_{k}$

$\rho \frac{\partial v_{k}}{\partial t}=\frac{\partial \sigma_{k l}}{\partial x_{l}}+\rho g_{k}$,

$D_{k l}=\delta_{k l}+\frac{\partial u_{k}}{\partial x_{l}^{0}}$

$\sigma_{k l}= \begin{cases}\frac{\lambda}{J} D_{k l} \operatorname{tr}\left(E_{k l}\right)+\frac{2 \mu}{J} D_{k l} E_{k l} & \text { for linear-elastic materials, } \\ \frac{\lambda \ln (J)}{J} \delta_{k l}+\frac{\mu}{J}\left(D_{k m} D_{l m}-\delta_{k l}\right) & \text { for neo-Hookean materials. }\end{cases}$

where $u_{k}$ is the displacement, $t$ is the time, $v_{k}$ is the velocity, $\rho$ is the density, $g_{k}$ is the body force, $D_{k l}$ is the deformation gradient, $\delta_{k l}$ is the Kronecker delta, $\sigma_{k l}$ is the stress tensor, $x^{0}$ is the position in the reference configuration, $\lambda$ is the Lamé constant, $E_{k l}$ is the strain tensor defined as $\frac{1}{2}\left(D_{k l}+D_{l k}\right)-\delta_{k l}$, $J$ is the determinant of the deformation gradient and $\mu$ is the shear modulus. Equations 2 and 4 describe the conservation of linear momentum and the material model, respectively.

Initial conditions are required for the displacement, velocity and stress tensor. The boundary of the domain $\Omega$ can be divided into a part with a Dirichlet boundary condition for the displacement and a part with a Neumann boundary condition for the traction:

$u_{k}(\boldsymbol{x}, t)=U_{k}(\boldsymbol{x}, t)$ on $\partial \Omega_{u}$,

$\sigma_{k l}(\boldsymbol{x}, t) n_{l}=\tau_{k}(\boldsymbol{x}, t)$ on $\partial \Omega_{\tau}$,

where $\boldsymbol{x}=\left[\begin{array}{ll}x_{1} & x_{2}\end{array}\right]^{T}$ and $\boldsymbol{n}$ is the unit vector normal to the boundary $\partial \Omega$ and pointing outwards. We remark that the domain $\Omega$, its boundary $\partial \Omega=\partial \Omega_{u} \cup \partial \Omega_{\tau}$ and the normal unit vector $\boldsymbol{n}$ may all depend on time. 
For solving the equations of motion in the Material Point Method, the conservation of linear momentum in Eq. 2 is needed in its weak form. For the weak form, Eq. 2 is first multiplied by a continuous test function $\phi$ that vanishes on $\partial \Omega_{u}$ and is subsequently integrated over the domain $\Omega$ :

$$
\int_{\Omega} \phi \rho a_{k} \mathrm{~d} \Omega=\int_{\Omega} \phi \frac{\partial \sigma_{k l}}{\partial x_{l}} \mathrm{~d} \Omega+\int_{\Omega} \phi \rho g_{k} \mathrm{~d} \Omega
$$

in which $a_{k}=\frac{\partial v_{k}}{\partial t}$ is the acceleration. After applying integration by parts, the Gauss integration theorem and splitting the boundary into Dirichlet and Neumann part, the weak formulation becomes

$$
\int_{\Omega} \phi \rho a_{k} \mathrm{~d} \Omega=\int_{\partial \Omega_{\tau}} \phi \tau_{k} \mathrm{~d} S-\int_{\Omega} \frac{\partial \phi}{\partial x_{l}} \sigma_{k l} \mathrm{~d} \Omega+\int_{\Omega} \phi \rho g_{k} \mathrm{~d} \Omega,
$$

whereby the contribution on $\partial \Omega_{u}$ equals zero, as the test function vanishes on this part of the boundary.

\subsection{Discretised equations}

Equation 8 can be solved using a finite element approach by defining $n_{\mathrm{bf}}$ basis functions $\phi_{i}\left(i=1, \ldots, n_{\mathrm{bf}}\right)$. The acceleration field $a_{k}$ is then discretised as a linear combination of these basis functions:

$a_{k}(\boldsymbol{x}, t)=\sum_{j=1}^{n_{\mathrm{bf}}} \hat{a}_{k, j} \phi_{j}(\boldsymbol{x})$

in which $\hat{a}_{k, j}$ is the time-dependent $j^{\text {th }}$ acceleration coefficient corresponding to basis function $\phi_{j}$. Substituting Eq. 9 into Eq. 8 and expanding the test function in terms of the basis functions $\phi_{i}$ leads to

$$
\begin{aligned}
& \int_{\Omega} \phi_{i} \rho\left(\sum_{j=1}^{n_{\mathrm{bf}}} \hat{a}_{k, j} \phi_{j}\right) \mathrm{d} \Omega \\
& =\int_{\partial \Omega_{\tau}} \phi_{i} \tau_{k} \mathrm{~d} S-\int_{\Omega} \frac{\partial \phi_{i}}{\partial x_{l}} \sigma_{k l} \mathrm{~d} \Omega+\int_{\Omega} \phi_{i} \rho g_{k} \mathrm{~d} \Omega,
\end{aligned}
$$

which holds for $i=1, \ldots, n_{\mathrm{bf}}$. By exchanging summation and integration, this can be rewritten in matrix-vector form as follows:

$$
\begin{aligned}
& \sum_{j=1}^{n_{\mathrm{bf}}} \underbrace{\left(\int_{\Omega} \phi_{i} \rho \phi_{j} \mathrm{~d} \Omega\right)}_{M_{i j}} \hat{a}_{k, j} \\
& =\underbrace{\int_{\partial \Omega_{\tau}} \phi_{i} \tau_{k} \mathrm{~d} S}_{F_{k, i}^{\text {trac }}}-\underbrace{\int_{\Omega} \frac{\partial \phi_{i}}{\partial x_{l}} \sigma_{k l} \mathrm{~d} \Omega}_{F_{k, i}^{\text {int }}}+\underbrace{\int_{\Omega} \phi_{i} \rho g_{k} \mathrm{~d} \Omega}_{F_{k, i}^{\text {body }}},
\end{aligned}
$$

$$
\Rightarrow \boldsymbol{M} \hat{\boldsymbol{a}}_{k}=\boldsymbol{F}_{k}^{\mathrm{trac}}-\boldsymbol{F}_{k}^{\mathrm{int}}+\boldsymbol{F}_{k}^{\mathrm{body}},
$$

where $\boldsymbol{M}$ denotes the mass matrix, $\hat{\boldsymbol{a}}_{k}$ the coefficient vector for the acceleration, while $\boldsymbol{F}_{k}^{\text {trac }}, \boldsymbol{F}_{k}^{\text {int }}$ and $\boldsymbol{F}_{k}^{\text {body }}$ denote, respectively, the traction, internal and body force vector in the $x_{k}$-direction. When density, stress and body force are known at time $t$, the coefficient vector $\hat{\boldsymbol{a}}_{k}$ is found from Eq. 12 . The properties of the continuum at time $t+\Delta t$ can then be determined using the acceleration field at time $t$.

\subsection{Solution procedure}

Within MPM, the continuum is discretised by a set of particles that store all its physical properties. At each time step, the particle information is projected onto a background grid, on which the momentum equation is solved. Particle properties and positions are updated according to this solution, as illustrated in Fig. 1.

To solve Eq. 12 at every time step, the integrals in Eq. 11 have to be evaluated. Since the material properties are only known at the particle positions, these positions are used as integration points for the numerical quadrature, and the particle volumes $V$ are adopted as integration weights. Assume that the total number of particles is equal to $n_{p}$. Here, subscript $p$ corresponds to particle properties. A superscript $t$ is assigned to particle properties that change over time. The mass matrix and force vectors are then defined by

$$
\begin{aligned}
& M_{i j}^{t}=\sum_{p=1}^{n_{p}} \underbrace{V_{p}^{t} \rho_{p}^{t}}_{m_{p}} \phi_{i}\left(\boldsymbol{x}_{p}^{t}\right) \phi_{j}\left(\boldsymbol{x}_{p}^{t}\right) \\
& F_{k, i}^{\mathrm{trac}, t}=\sum_{p=1}^{n_{p}} \phi_{i}\left(\boldsymbol{x}_{p}^{t}\right) f_{k, p}^{\mathrm{trac}, t} \\
& F_{k, i}^{\mathrm{int}, t}=\sum_{p=1}^{n_{p}} V_{p}^{t} \frac{\partial \phi_{i}}{\partial x_{m}}\left(\boldsymbol{x}_{p}^{t}\right) \sigma_{m k, p}^{t} \\
& F_{k, i}^{\mathrm{body}, t}=\sum_{p=1}^{n_{p}} \underbrace{V_{p}^{t} \rho_{p}^{t}}_{m_{p}} \phi_{i}\left(\boldsymbol{x}_{p}^{t}\right) g_{k}
\end{aligned}
$$

in which $m_{p}$ denotes the particle mass, which is set to remain constant over time, guaranteeing conservation of the total mass. The coefficient vector $\hat{\boldsymbol{a}}_{k}^{t}$ at time $t$ for the $x_{k}$-direction is determined by solving

$$
\boldsymbol{M}^{t} \hat{\boldsymbol{a}}_{k}^{t}=\left(\boldsymbol{F}_{k}^{\mathrm{trac}, t}-\boldsymbol{F}_{k}^{\mathrm{int}, t}+\boldsymbol{F}_{k}^{\mathrm{body}, t}\right)
$$

Unless otherwise stated, Eq. 17 is solved adopting the consistent mass matrix. Next, the reconstructed acceleration field is used to update the particle velocity: 


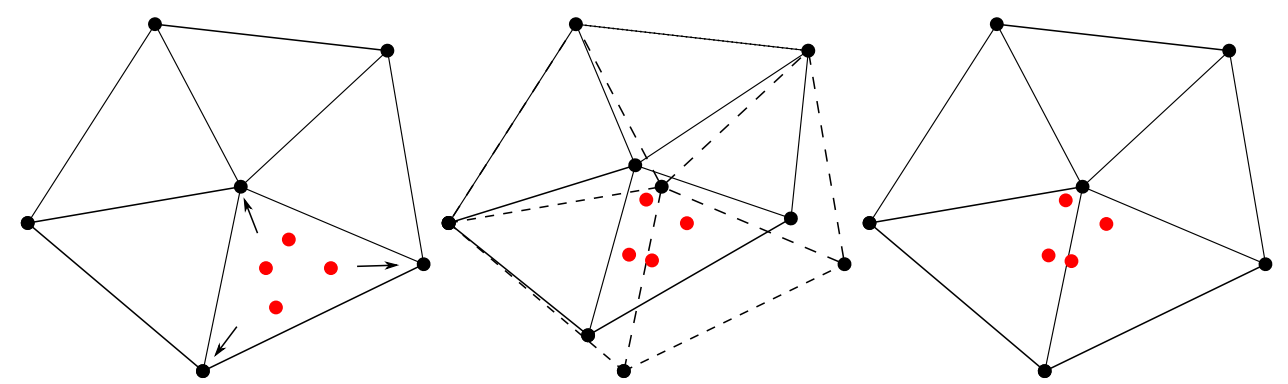

Fig. 1 One time step in the MPM procedure. Projection of particle properties onto the basis functions of the background grid (left). Update of particle properties based on the solution on the background grid (middle). Resetting the grid (right)

$v_{k, p}^{t+\Delta t}=v_{k, p}^{t}+\Delta t a_{k}^{t}\left(\boldsymbol{x}_{p}^{t}\right)=v_{k, p}^{t}+\Delta t \sum_{j=1}^{n_{\mathrm{bf}}} \hat{a}_{k, j}^{t} \phi_{j}\left(\boldsymbol{x}_{p}^{t}\right)$

The semi-implicit Euler-Cromer scheme [5] is adopted to update the remaining particle properties. First, the velocity field $v_{k}^{t+\Delta t}$ is discretised as a linear combination of the same basis functions, similar to the acceleration field (Eq. 9):

$v_{k}^{t+\Delta t}(\boldsymbol{x})=\sum_{j=1}^{n_{\mathrm{bf}}} \hat{v}_{k, j}^{t+\Delta t} \phi_{j}(\boldsymbol{x})$,

in which $\hat{\boldsymbol{v}}_{k}^{t+\Delta t}$ is the velocity coefficient vector for the velocity field at time $t+\Delta t$ in the $x_{k}$-direction. The coefficient vector $\hat{\boldsymbol{v}}_{k}^{t+\Delta t}$ is then determined from a density weighted $L_{2-}$ projection onto the basis functions $\phi_{i}$. This results in the following system of equations:

$\boldsymbol{M}^{t} \hat{\boldsymbol{v}}_{k}^{t+\Delta t}=\boldsymbol{P}_{k}^{t+\Delta t}$,

where $\boldsymbol{M}^{t}$ is the same mass matrix as defined in Eq. 13 . $\boldsymbol{P}_{k}^{t+\Delta t}$ denotes the momentum vector with the coefficients given by

$P_{k, i}^{t+\Delta t}=\sum_{p=1}^{n_{p}} \underbrace{V_{p}^{t} \rho_{p}^{t}}_{m_{p}} v_{k, p}^{t+\Delta t} \phi_{i}\left(\boldsymbol{x}_{p}\right)$.

Particle properties are subsequently updated in correspondence with Eqs. 1-4. First, the deformation gradient and its determinant are obtained:

$\boldsymbol{D}_{p}^{t+\Delta t}=\left(\boldsymbol{I}+\boldsymbol{\varepsilon}_{p}^{t+\Delta t} \Delta t\right) \boldsymbol{D}_{p}^{t}$,

$J_{p}^{t+\Delta t}=\operatorname{det}\left(\boldsymbol{D}_{p}^{t+\Delta t}\right)$.

Here, $\boldsymbol{I}$ denotes the identify matrix and $\boldsymbol{\varepsilon}_{p}^{t+\Delta t}$ denotes the symmetric part of the velocity gradient: $\varepsilon_{k l, p}^{t+\Delta t}=\frac{1}{2} \sum_{j=1}^{n_{\mathrm{bf}}}\left(\hat{v}_{k, j}^{t+\Delta t} \frac{\partial \phi_{j}}{\partial x_{l}}\left(\boldsymbol{x}_{p}^{t}\right)+\hat{v}_{l, j}^{t+\Delta t} \frac{\partial \phi_{j}}{\partial x_{k}}\left(\boldsymbol{x}_{p}^{t}\right)\right)$.

For linear-elastic materials, the particle stresses are computed as follows:

$E_{k l, p}^{t+\Delta t}=\frac{1}{2}\left(D_{k l, p}^{t+\Delta t}+D_{l k, p}^{t+\Delta t}\right)-\delta_{k l}$,

$\sigma_{k l, p}^{t+\Delta t}=\lambda \delta_{k l} \operatorname{tr}\left(E_{k l, p}^{t+\Delta t}\right)+2 \mu E_{k l, p}^{t+\Delta t}$.

For neo-Hookean materials, the stresses are obtained from

$\sigma_{k l, p}^{t+\Delta t}=\frac{\lambda \ln \left(J_{p}^{t+\Delta t}\right)}{J_{p}^{t+\Delta t}} \delta_{k l}+\frac{\mu}{J_{p}^{t+\Delta t}}\left(D_{k m, p}^{t+\Delta t} D_{l m, p}^{t+\Delta t}-\delta_{k l}\right)$.

The determinant of the deformation gradient is used to update the volume of each particle. Based on this volume, the density is updated in such way that the mass of each particle remains constant:

$V_{p}^{t+\Delta t}=J_{p}^{t+\Delta t} V_{p}^{0}$,

$\rho_{p}^{t+\Delta t}=m_{p} / V_{p}^{t+\Delta t}$.

Finally, particle positions and displacements are updated from the velocity field:

$x_{k, p}^{t+\Delta t}=x_{k, p}^{t}+\Delta t \sum_{i=1}^{n_{\mathrm{bf}}} v_{k, i}^{t+\Delta t} \phi_{i}\left(\boldsymbol{x}_{p}^{t}\right)$

$u_{k, p}^{t+\Delta t}=u_{k, p}^{t}+\Delta t \sum_{i=1}^{n_{\mathrm{bf}}} v_{k, i}^{t+\Delta t} \phi_{i}\left(\boldsymbol{x}_{p}^{t}\right)$.

The described MPM can be numerically implemented by performing the steps in Eqs. 13-31 in each time step in the shown order. Note that all steps can be applied with a variety of basis functions, without any essential difference in the algorithm. In this paper, we investigate the use of PowellSabin spline basis functions, which are described in the next section. 
Fig. 2 A triangular grid (left) and a possible PS-refinement (right). The molecule $\Omega_{i}$ around vertex $V_{i}$ is marked grey. (Color figure online)
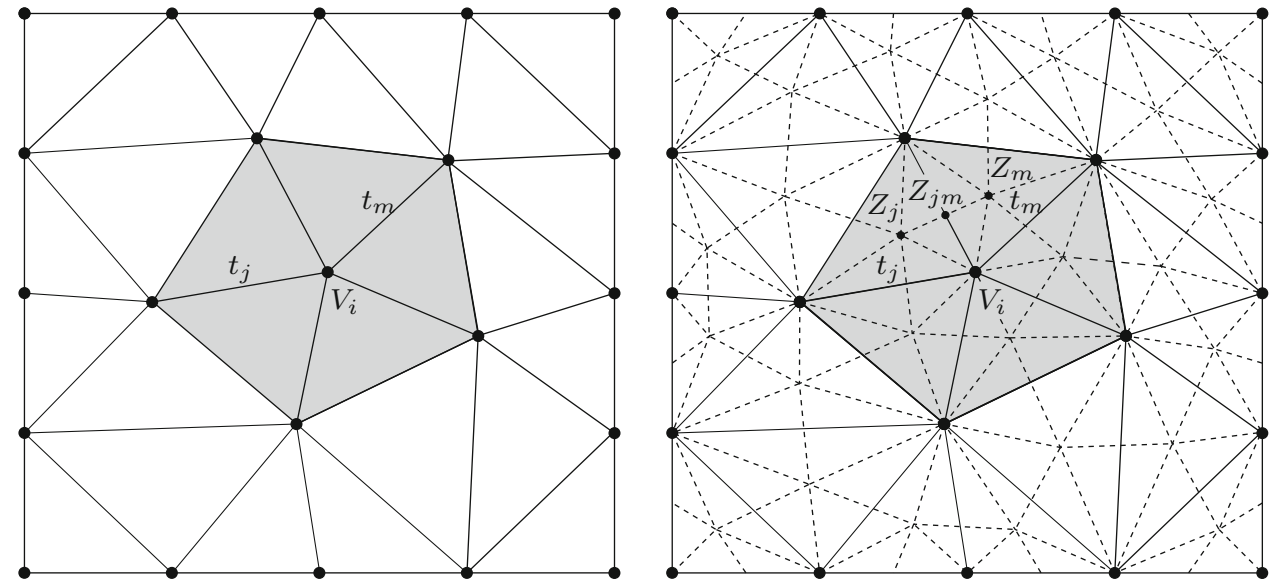
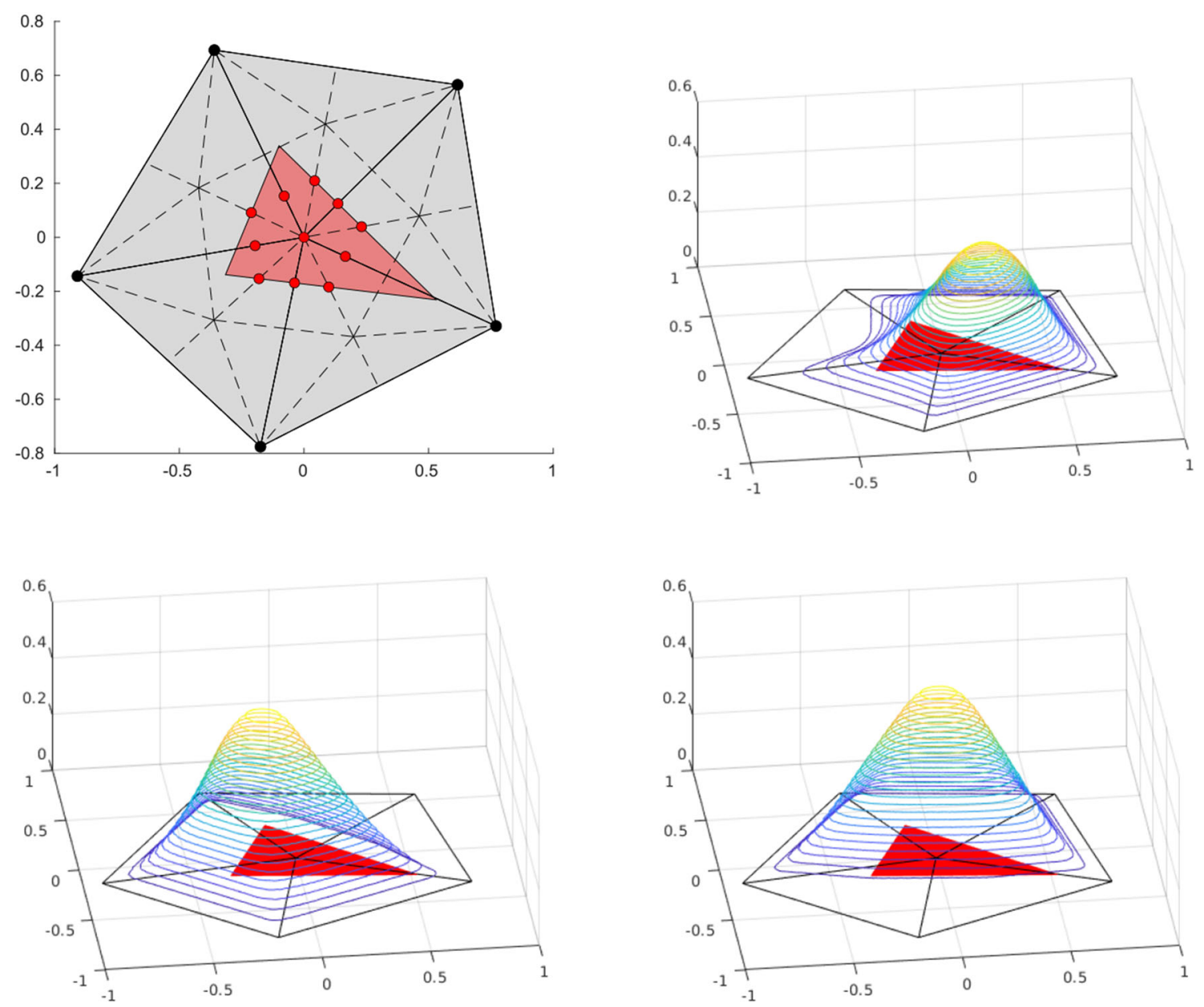

Fig. 3 Top left: the PS-points of the middle vertex, marked with red dots, and a possible control triangle containing all PS-points. Other subfigures: the three PS-splines associated with this control triangle. (Color figure online)

\section{Powell-Sabin spline basis functions}

In this section, the construction and essential properties of Powell-Sabin (PS) spline basis functions are summarised. For the full construction process and the proofs of the properties, we refer to the publication by Dierckx $[8,9]$. The considered PS-splines are piecewise quadratic splines with global $C^{1}$-continuity. They are defined on arbitrary triangulations, have local support and possess the properties of non-negativity and partition of unity. Several examples of PS-splines are shown in Fig. 3. 


\subsection{Powell-Sabin grid refinement}

To construct PS-splines on an arbitrary triangulation, a Powell-Sabin refinement is required, dividing each of the original main-triangles into six sub-triangles as follows (see also Fig. 2).

1. For each triangle $t_{j}$, choose an interior point $Z_{j}$ (e.g. the incentre), such that if triangles $t_{j}$ and $t_{m}$ have a common edge, the line between $Z_{j}$ and $Z_{m}$ intersects the edge. The intersection point is called $Z_{j m}$.

2. Connect each $Z_{j}$ to the vertices of its triangle $t_{j}$ with straight lines.

3. Connect each $Z_{j}$ to all edge points $Z_{j m}$ with straight lines. In case $t_{j}$ is a boundary element, connect $Z_{j}$ to an arbitrary point on each boundary edge (e.g. the edge middle).

The area consisting of the main-triangles around $V_{i}$ is referred to as the molecule $\Omega_{i}$ of $V_{i}$ (see Fig. 2). Each PSspline is associated with a main vertex $V_{i}$ and will be defined on the molecule $\Omega_{i}$.

\subsection{Control triangles}

For each main vertex $V_{i}$, the set of PS-points is given by the union of $V_{i}$ and the sub-triangle edge midpoints directly around it, see Fig. 3. A control triangle for $V_{i}$ is then defined as a triangle that contains all its PS-points. Note that this control triangle is not uniquely defined. However, it preferably has a small area to ensure good stability properties of the resulting PS-splines [8].

A sufficiently good control triangle can be constructed by considering only control triangles with two or three edges shared with the convex hull of the PS-points and taking the one with smallest surface. Further details about the implementation of such an algorithm can be found in [6].

Each control triangle defines three basis functions, all associated with vertex $V_{i}$ and the molecule $\Omega_{i}$. Therefore, the basis functions are indexed $\phi_{i}^{q}\left(i=1, \ldots, n_{v}, q=1,2,3\right)$, where $i$ indicates the vertex $V_{i}, n_{v}$ the number of vertices and $q$ the local index. Each basis function $\phi_{i}^{q}$ is uniquely defined by a triplet $\left(\alpha_{i}^{q}, \beta_{i}^{q}, \gamma_{i}^{q}\right)$, containing the value and gradient of $\phi_{i}^{q}$ in its associated vertex $V_{i}$,

$\left(\alpha_{i}^{q}, \beta_{i}^{q}, \gamma_{i}^{q}\right)=\left(\phi_{i}^{q}\left(V_{i}\right), \frac{\partial \phi_{i}^{q}}{\partial x}\left(V_{i}\right), \frac{\partial \phi_{i}^{q}}{\partial y}\left(V_{i}\right)\right)$.

The triplets of the three PS-spline basis functions corresponding to vertex $V_{i}$ are determined from the control triangle and the position of $V_{i}$. Let the Cartesian coordinates of the control triangle vertices be $Q_{i}^{1}=\left(X_{i}^{1}, Y_{i}^{1}\right), Q_{i}^{2}=\left(X_{i}^{2}, Y_{i}^{2}\right)$

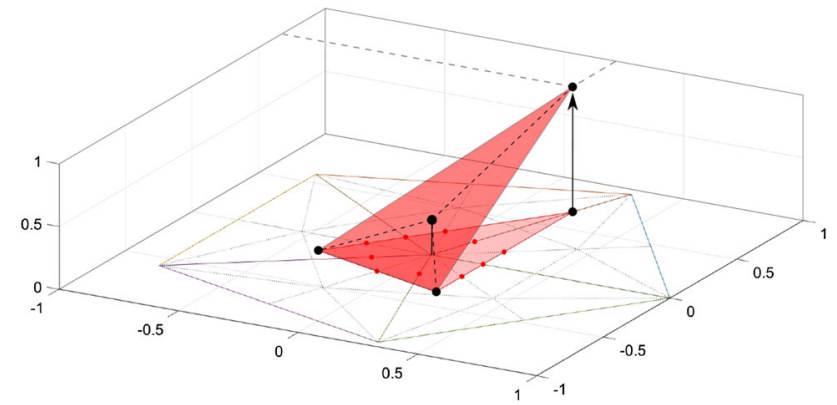

Fig. 4 A control triangle lifted to $z=1$ in one of its vertices for determining a triplet of a basis function. The location in the lifted plane above the vertex $V_{i}$ in the middle is marked with a dot; the value and gradient there correspond to the triplet of the associated basis function

and $Q_{i}^{3}=\left(X_{i}^{3}, Y_{i}^{3}\right)$, and the Cartesian coordinates of $V_{i}=$ $\left(x_{i}, y_{i}\right)$. The three triplets are then determined by solving

$$
\left[\begin{array}{ccc}
X_{i}^{1} & X_{i}^{2} & X_{i}^{3} \\
Y_{i}^{1} & Y_{i}^{2} & Y_{i}^{3} \\
1 & 1 & 1
\end{array}\right]\left[\begin{array}{ccc}
\alpha_{i}^{1} & \beta_{i}^{1} & \gamma_{i}^{1} \\
\alpha_{i}^{2} & \beta_{i}^{2} & \gamma_{i}^{2} \\
\alpha_{i}^{3} & \beta_{i}^{3} & \gamma_{i}^{3}
\end{array}\right]=\left[\begin{array}{ccc}
x_{i} & 1 & 0 \\
y_{i} & 0 & 1 \\
1 & 0 & 0
\end{array}\right]
$$

The control triangle has a direct geometric interpretation for the triplet. Solving for the triplet $\left(\alpha_{i}^{q}, \beta_{i}^{q}, \gamma_{i}^{q}\right)$ of a basis function $\phi_{i}^{q}$ corresponds geometrically to 'lifting' up control triangle vertex $Q_{i}^{q}$ of the control triangle in the $z$-direction to 1 , and evaluating the height and gradient of the resulting plane at the location of $V_{i}$, as shown Fig. 4.

\subsection{Bernstein-Bézier formulation for quadratic splines}

From the triplet $\left(\alpha_{i}^{q}, \beta_{i}^{q}, \gamma_{i}^{q}\right)$, the PS-spline $\phi_{i}^{q}$ is constructed as a piecewise quadratic polynomial over each sub-triangle in the PS-refinement in barycentric coordinates. Cartesian coordinates $(x, y)$ can be converted to barycentric coordinates $\left(\eta_{1}, \eta_{2}, \eta_{3}\right)$ with respect to the considered triangle vertices $\left(x_{1}, y_{1}\right),\left(x_{2}, y_{2}\right),\left(x_{3}, y_{3}\right)$ as follows (see also Fig. 5, left):

$\left[\begin{array}{ccc}x_{1} & x_{2} & x_{3} \\ y_{1} & y_{2} & y_{3} \\ 1 & 1 & 1\end{array}\right]\left[\begin{array}{l}\eta_{1} \\ \eta_{2} \\ \eta_{3}\end{array}\right]=\left[\begin{array}{l}x \\ y \\ 1\end{array}\right]$.

Next, we define 6 quadratic Bernstein polynomials $B_{i, j, k}$ for each sub-triangle in barycentric coordinates,

$B_{i, j, k}(\eta)=\frac{2 !}{i ! j ! k !} \eta_{1}^{i} \eta_{2}^{j} \eta_{3}^{k}$, with $i, j, k \in\{0,1,2\}$,

and $i+j+k=2$,

which are non-negative and form a partition of unity. Any desired quadratic polynomial $b(\boldsymbol{\eta})$ can be uniquely 
Fig. 5 Barycentric coordinates with respect to the sub-triangle vertices (left) and the Bézier ordinates of a quadratic polynomial $b(\boldsymbol{\eta})$ (right), schematically represented at the same location
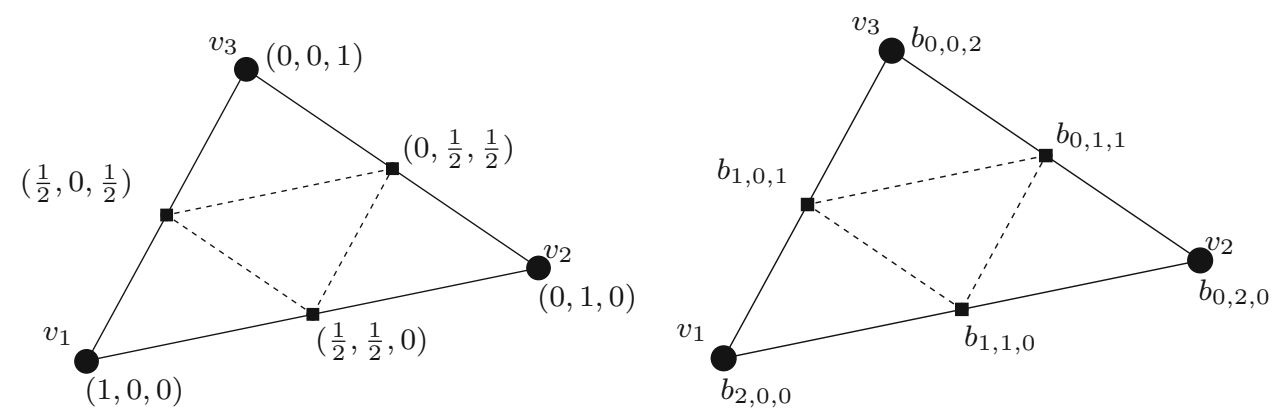

Fig. 6 The molecule around a vertex $V_{i}$ (left) and one of the main-triangles in the molecule (right). The locations of the middle vertex $Z$ and the edge vertices $R$ are marked with respect to the vertices $v_{1}, v_{2}$ and $v_{3} . v_{1}\left(=V_{i}\right)$ is also marked with the barycentric coordinates with respect to the control triangle

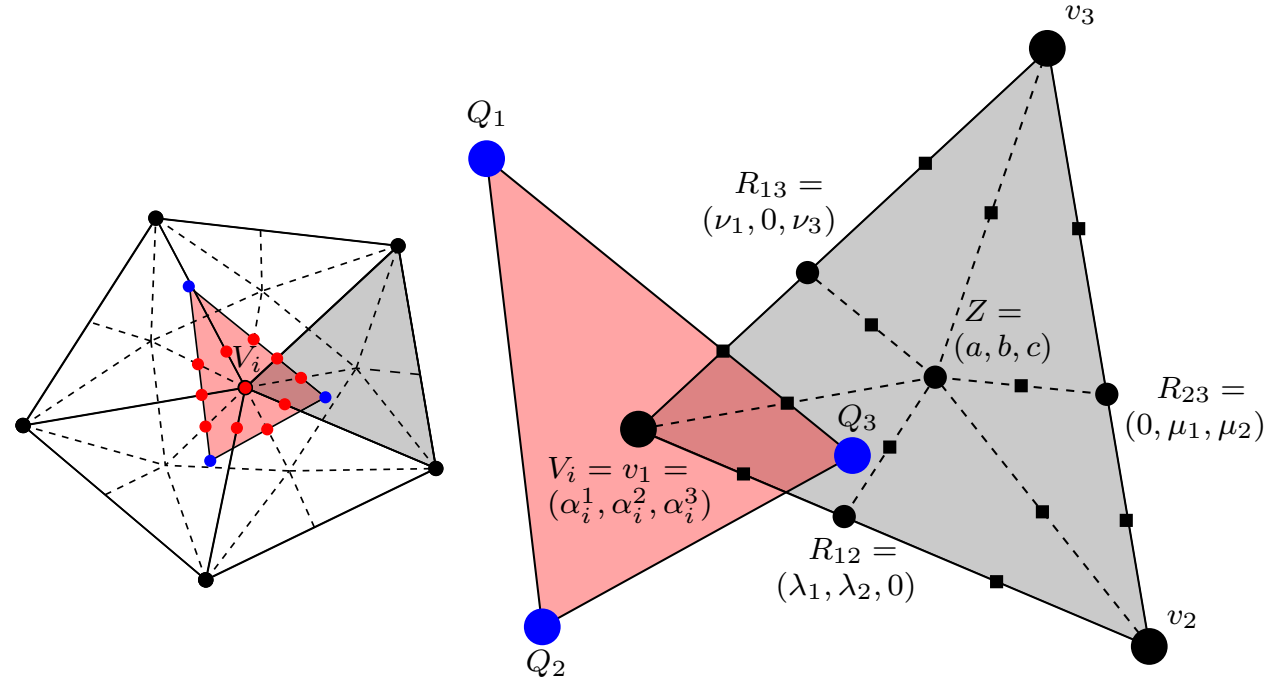

constructed as a linear combination of the 6 quadratic Bernstein polynomials $B_{i, j, k}$ over a sub-triangle,

$b(\boldsymbol{\eta})=\sum_{\substack{i+j+k=2, i, j, k \in\{0,1,2\}}} b_{i, j, k} B_{i, j, k}(\boldsymbol{\eta})$,

where $b_{i, j, k}$ are called the Bézier ordinates. The desired quadratic polynomial can thus be fully defined by its Bézier ordinates, which can be schematically represented by associating Bézier ordinate $b_{i, j, k}$ with barycentric coordinates $\left(\frac{i}{2}, \frac{j}{2}, \frac{k}{2}\right)$, as shown in Fig. 5 .

\subsection{Construction of PS-splines}

Next, consider the molecule $\Omega_{i}$ around a vertex $V_{i}$ and one of the main-triangles $t_{j}$ in the molecule, as depicted in Fig. 6. The grey main-triangle has been divided into 6 sub-triangles in the PS-refinement. In each of the sub-triangles, the 6 locations with barycentric coordinates $\left(\frac{i}{2}, \frac{j}{2}, \frac{k}{2}\right), i+j+k=$ $2, i, j, k \in\{0,1,2\}$ have been marked. Denote the vertices of the main-triangle by $v_{1}, v_{2}$ and $v_{3}$, with $v_{1}\left(=V_{i}\right)$ the middle vertex of the considered molecule, and $v_{2}$ and $v_{3}$ be indexed counter-clockwise. Let the centre vertex, $Z$, of the grey triangle has barycentric coordinates $Z=(a, b, c)$ with respect to vertices $v_{1}, v_{2}$ and $v_{3}$. Furthermore, let the edge vertices $R$ have barycentric coordinates $R_{12}=\left(\lambda_{1}, \lambda_{2}, 0\right)$, $R_{23}=\left(0, \mu_{2}, \mu_{3}\right)$ and $R_{13}=\left(v_{1}, 0, v_{3}\right)$ with respect to $v_{1}$, $v_{2}$ and $v_{3}$, as denoted in Fig. 6. Finally, let $v_{1}$ have barycentric coordinates $v_{1}=\left(\alpha_{i}^{1}, \alpha_{i}^{2}, \alpha_{i}^{3}\right)$ with respect to the control triangle vertices $Q_{i}^{1}, Q_{i}^{2}$ and $Q_{i}^{3}$. These values of $\alpha$ also correspond to the first values of the three triplets of the basis functions associated with this molecule, see Equation (33).

Finally, the three basis function $\phi_{i}^{q}, q=1,2,3$, associated with vertex $V_{i}$, are defined by their Bézier ordinates on each of the sub-triangles within the molecule of $V_{i}$. The Bézier ordinates of a basis function $\phi_{i}^{q}$ are shown in Fig. 7 . The coefficients in the figure are given in terms of the triplet $(\alpha, \beta, \gamma)=\left(\alpha_{i}^{q}, \beta_{i}^{q}, \gamma_{i}^{q}\right)$ of the considered basis function $\phi_{i}^{q}$ and the barycentric coordinates of $Z=(a, b, c), R_{12}=$ $\left(\lambda_{1}, \lambda_{2}, 0\right), R_{13}=\left(v_{1}, 0, v_{3}\right)$, and the Cartesian coordinates of the considered triangle $v_{1}=\left(x_{1}, y_{1}\right), v_{2}=\left(x_{2}, y_{2}\right)$ and $v_{3}=\left(x_{3}, y_{3}\right)$ as follows, 


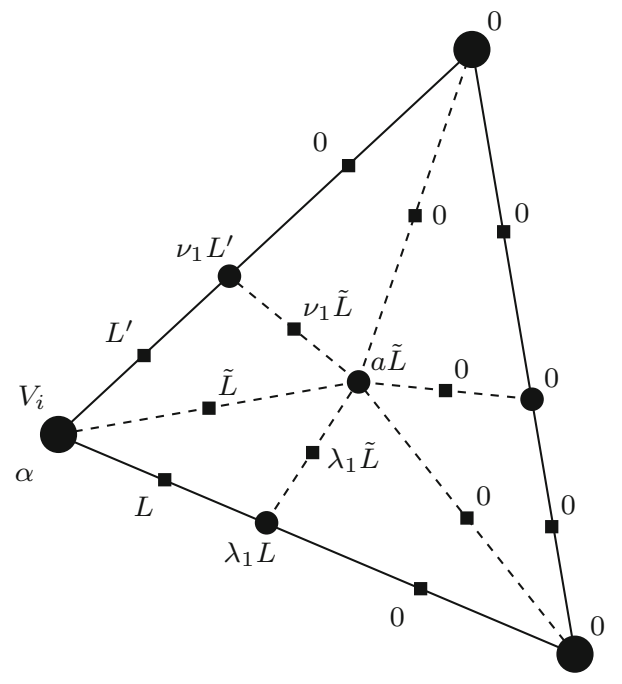

Fig. 7 The Bézier ordinates for basis function $\phi_{i}^{1}$, corresponding to the vertex $V_{i}$ considered in Fig. 6 . There are three different basis functions, note that for each of these basis functions, $\alpha, \beta$ and $\gamma$ should correspond with the triplet $\left(\alpha^{q}, \beta^{q}, \gamma^{q}\right)$ corresponding to PS-triangle vertex $Q_{q}$

$L=\alpha+\frac{1-\lambda_{1}}{2} \bar{\beta}$,

$L^{\prime}=\alpha+\frac{1-v_{1}}{2} \bar{\gamma}$,

$\tilde{L}=\alpha+\frac{b}{2} \bar{\beta}+\frac{c}{2} \bar{\gamma}$,

with

$\bar{\beta}=\beta\left(x_{2}-x_{1}\right)+\gamma\left(y_{2}-y_{1}\right)$ and

$\bar{\gamma}=\beta\left(x_{3}-x_{1}\right)+\gamma\left(y_{3}-y_{1}\right)$.

The resulting PS-splines corresponding to the shown control triangle of vertex $V_{i}$ are shown on the associated molecule in Fig. 3.

\subsection{Projecting on PS-splines and lumping within PS-MPM}

In this section, we show by numerical experiments that the projection on PS-splines leads to third-order spatial convergence in the $L_{2}$-error between an analytic function and its projection. Furthermore, we also investigate the effect of performing a projection on PS-splines using a lumped variant of the mass matrix instead of the consistent one. Lumping of the mass matrix is often used in MPM with piecewise-linear basis functions, but we will show that lumping is less suitable for MPM with PS-spline basis function when no special measures are taken.

We consider the projection of a two-dimensional sine function onto a basis of PS-splines, using a consistent and a lumped mass matrix, respectively. The results are also com- pared with a projection onto a basis of piecewise-linear basis functions. We project the function $f=\sin (x / \pi) \sin (y / \pi)$ on the unit square onto a basis of PS-splines using a standard $L_{2}$-projection:

$$
\begin{aligned}
& \hat{f}(x, y)=\sum_{j=1}^{n_{\text {bf }}} c_{j} \phi_{j}(x, y) ; \\
& \sum_{j=1}^{n_{\text {bf }}} \underbrace{\left(\int_{\Omega} \phi_{i} \phi_{j} \mathrm{~d} \Omega\right)}_{M_{i j}} c_{j}=\underbrace{\int_{\Omega} \phi_{i} f \mathrm{~d} \Omega}_{b_{i}} .
\end{aligned}
$$

The entries of the mass matrix $\boldsymbol{M}$ and the right-hand side $\boldsymbol{b}$ are evaluated using high-order Gauss integration such that the integration error is insignificant. Finally, we may obtain the projection $\hat{f}$ by either solving $\boldsymbol{M c}=\boldsymbol{b}$ consistently, or by using the lumped mass matrix, $b_{i}=c_{i} / \tilde{M}_{i i}$, in which the lumped mass matrix $\tilde{M}$ has been created by lumping all the mass of each row onto its diagonal element, $\tilde{M}_{i i}=$ $\sum_{j=1}^{n_{\mathrm{bf}}} M_{i j}, \tilde{M}_{i j}=0$ for $i \neq j$. A basis of PS-splines, and of piecewise-linear functions were adopted, respectively, both defined on a structured triangulation.

Figure 8 shows the $L_{2}$-error in the function value and $x$ derivative for various refinements of the grid. When using a consistent mass matrix, the expected orders of convergence are obtained for piecewise-linear basis functions and PS-spline basis functions, both in the function value and the $x$-derivative. The use of a lumped mass matrix leads to a firstorder accurate approximation of the function value for both types of basis functions. However, the $x$-derivative does not converge at all when adopting a lumped mass matrix within a PS-spline basis. As the gradient of the reconstructed field is often used in MPM (e.g. Eq. 24), lumping within PS-MPM will often lead to spatial oscillations as will be shown in more detail in Sect. 4.3.

\section{Numerical results}

To validate the proposed PS-MPM, several benchmarks involving large deformations are considered. The first benchmark describes a thin vibrating bar, where the displacement is caused by an initial velocity field. For this benchmark, PS-MPM on a structured triangular grid is compared with a reference solution. A second benchmark considers a unit square undergoing axis-aligned displacement with known analytical solution. The spatial convergence of PS-MPM on an unstructured grid is determined for this benchmark. The third benchmark describes a solid column under self-weight. In this benchmark, we investigate the use of a lumped mass matrix in PS-MPM to stabilise the simulation when part of the original domain becomes empty. We will show that a 
Fig. 8 Error in the function value (left) and $x$-derivative (right) when projecting onto a basis of PS-splines (PS) and using a consistent (cons.) and a lumped mass matrix piecewise-linear functions (PL),
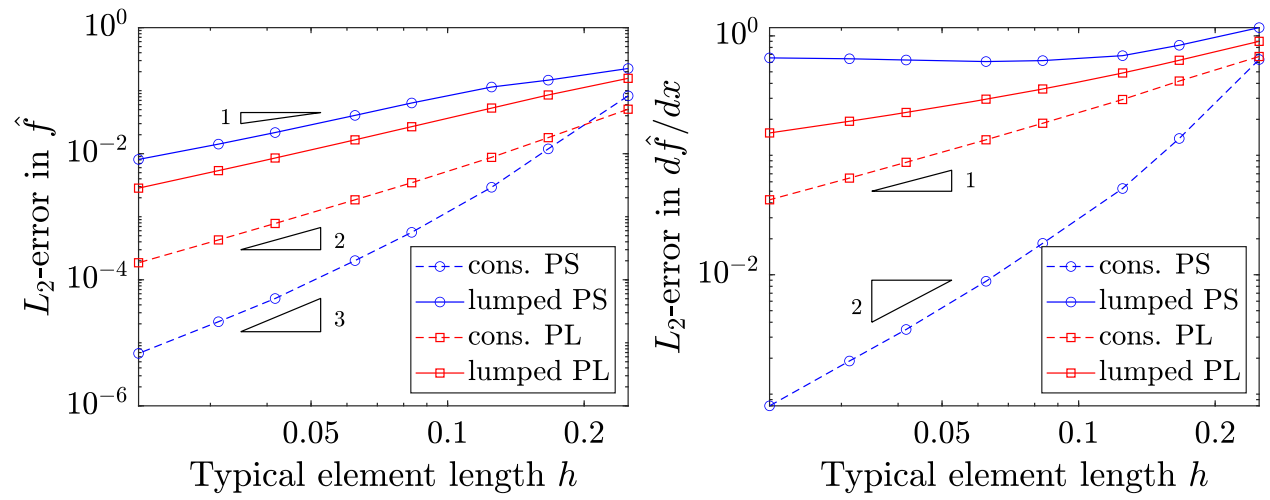

Fig. 9 The vibrating bar benchmark (top) and the initial particle configuration (bottom)

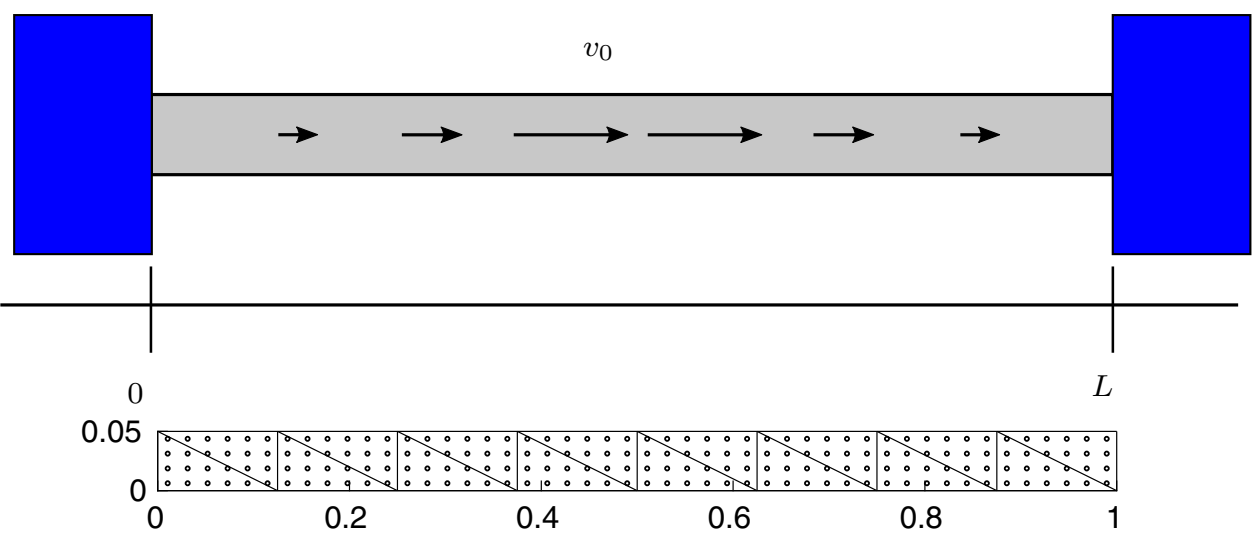

fully lumped mass matrix within PS-MPM leads to spatial oscillations, as expected from Sect. 3.5. Instead, we propose the use of a partially lumped mass matrix to mitigate these oscillations. Throughout this section, coordinates in the current configuration are denoted by $(x, y)$ and in the reference configuration by $\left(x^{0}, y^{0}\right)$.

\subsection{Vibrating bar}

In this section, a thin linear-elastic vibrating bar is considered with both ends fixed. A one-dimensional UL-FEM [36] solution on a very fine grid serves as reference. The grid used for PS-MPM and the initial particle positions are shown in Fig. 9.

The bar is modelled with density $\rho=25 \mathrm{~kg} / \mathrm{m}^{3}$, Young's modulus $E=50 \mathrm{~Pa}$, Poisson ratio $v=0$, length $L=1 \mathrm{~m}$, width $W=0.05 \mathrm{~m}$ and initial maximum velocity of $0.1 \mathrm{~m} / \mathrm{s}$. The chosen parameters result in a maximum normal strain in the $x$-direction of approximately $7 \%$. At the left and right boundary, homogeneous Dirichlet boundary conditions are imposed for both $x$ - and $y$-displacement, whereas at the top and bottom boundary, a homogeneous Dirichlet boundary condition is imposed only for the $y$-displacement, and a free-slip boundary condition for the $x$-direction. The initial displacement equals zero, but an initial $x$-velocity profile is set to $v_{x}\left(x^{0}, y^{0}\right)=0.1 \sin \left(x^{0} \pi / L\right)$. The initial $y$-velocity is equal to zero.

The time step size for the simulation is $\Delta t=5 \cdot 10^{-3} \mathrm{~s}$. The Courant number for two-dimensional problems is defined as $C=\frac{2 \Delta t}{h} \sqrt{E / \rho}$, in which $h$ is the typical element length, and $\sqrt{E / \rho}$ is the characteristic wave speed. Due to the ambiguity of $h$ for a PS-refinement on an unstructured triangular grid, we estimate the average edge length of the sub-triangles by $h \approx 0.025 \mathrm{~m}$. In this case, the Courant number is $C \approx 0.56<$ 1, satisfying the CFL condition [4].

Figure 10 shows the displacement in the middle of the bar over time and the stress profile through the entire bar at the end of the simulation. Although a relatively coarse PS-MPM grid and few particle are used, the method yields accurate results and a smooth stress profile.

In case of small and large deformations, the energy in the system over time is shown in Fig. 11. Results for small deformations have been obtained by setting the initial maximum velocity equal to $0.001 \mathrm{~m} / \mathrm{s}$. In the limit of small deformations, the PS-MPM solution is described exactly by a harmonic oscillator, and the simulation with small deformations is indeed in good agreement with the (sampled) exact solution. For large deformations, however, the solution is no longer perfectly periodic. Nonetheless, the energy over time obtained with PS-MPM strongly resembles the UL-FEM reference solution. Only after $7 \mathrm{~s}$, the PS-MPM simulation 
Fig. 10 Displacement of the particle initially positioned at $\left(x^{0}, y^{0}\right) \approx(0.5 \mathrm{~m}, 0.025 \mathrm{~m})$ over time (left) and the stress component $\sigma_{x x}$ along the bar at $t=2.5 \mathrm{~s}$ (right)

Fig. 11 Energy in the vibrating bar with large deformations (above), and for small deformations (below). Both are compared with a sampled reference solution
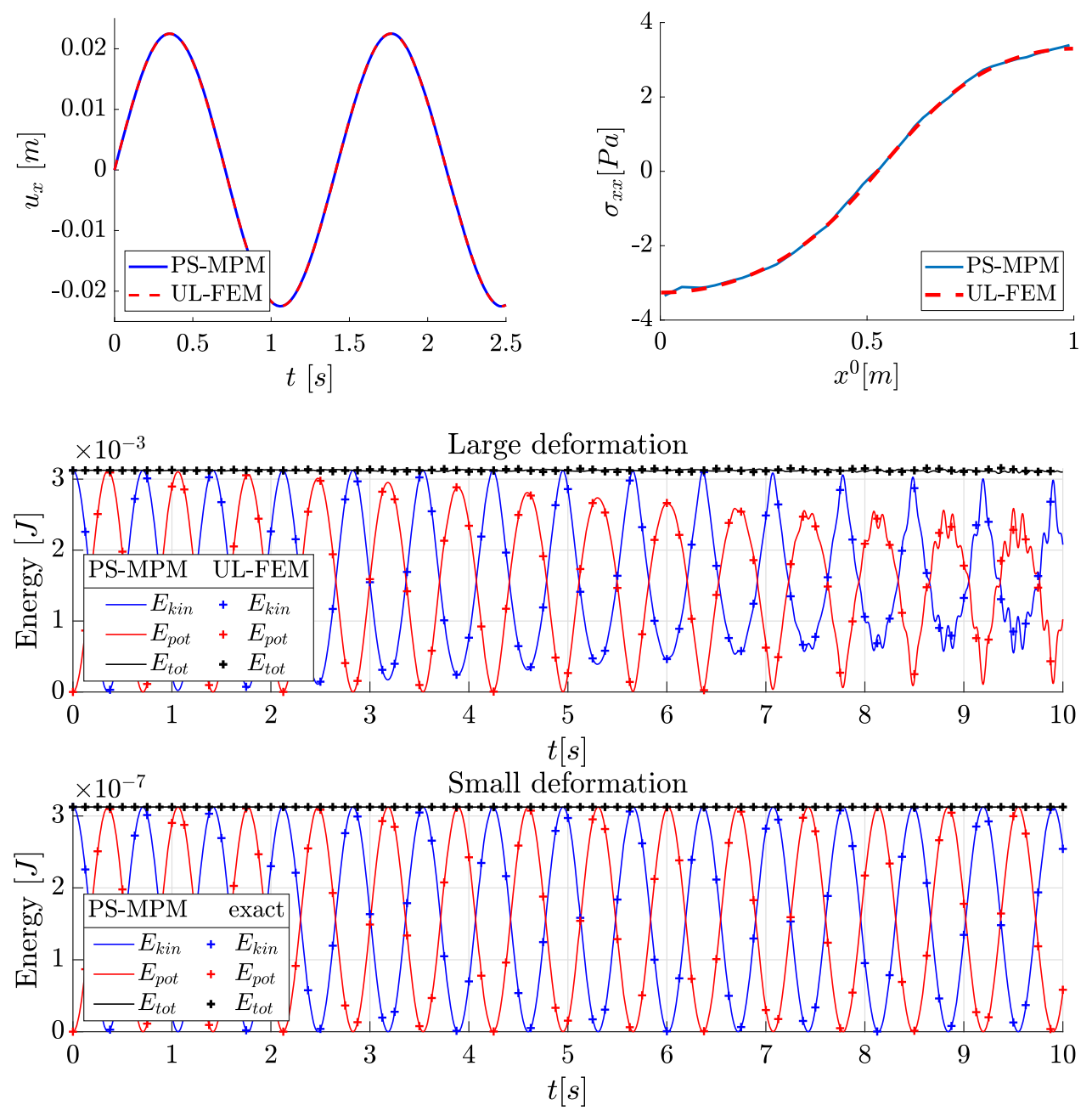

shows oscillations in the energy which are not present with UL-FEM. A visible decay in total energy due to numerical dissipation is only observed for simulation times larger than $15 \mathrm{~s}$.

\subsection{Axis-aligned displacement on an unstructured grid}

In this section, we consider two-dimensional axis-aligned displacement on the unit square $(L \times W$, with $L=W=1$ $m)$ to investigate the grid-crossing error and spatial convergence of PS-MPM on an unstructured grid. An analytical solution for this problem is constructed using the method of manufactured solutions: the analytical solution is assumed $a$ priori, and the corresponding body force is calculated accordingly. This benchmark has been adopted from [25] and [38]. The analytical solution for the displacement in terms of the reference configuration is given by

$u_{x}=u^{0} \sin \left(2 \pi x^{0}\right) \sin \left(\sqrt{E / \rho^{0}} \pi t\right)$, $u_{y}=u^{0} \sin \left(2 \pi y^{0}\right) \sin \left(\sqrt{E / \rho^{0}} \pi t+\pi\right)$.

Here, $\rho^{0}=10^{3} \mathrm{~kg} / \mathrm{m}^{3}, u^{0}=0.05 \mathrm{~m}$ and Young's modulus $E=10^{7} \mathrm{~Pa}$. The corresponding body forces [25] are

$g_{x}=\pi^{2} u_{x}\left(\frac{4 \mu}{\rho^{0}}-\frac{E}{\rho^{0}}-4 \frac{\lambda\left[\ln \left(D_{x x} D_{y y}\right)-1\right]-\mu}{\rho^{0} D_{x x}^{2}}\right)$,

$g_{y}=\pi^{2} u_{y}\left(\frac{4 \mu}{\rho^{0}}-\frac{E}{\rho^{0}}-4 \frac{\lambda\left[\ln \left(D_{x x} D_{y y}\right)-1\right]-\mu}{\rho^{0} D_{y y}^{2}}\right)$,

in which the Lamé constant $\lambda$, the shear modulus $\mu$ and the normal components of the deformation gradient $D_{x x}$ and $D_{y y}$ are defined as

$$
\begin{aligned}
& \lambda=\frac{E v}{(1+v)(1-2 v)}, \quad \mu=\frac{E}{2(1+v)}, \\
& D_{x x}=1+2 u^{0} \pi \cos \left(2 \pi x^{0}\right) \sin \left(\sqrt{E / \rho^{0}} \pi t\right),
\end{aligned}
$$


$D_{y y}=1+2 u^{0} \pi \cos \left(2 \pi y^{0}\right) \sin \left(\sqrt{E / \rho^{0}} \pi t+\pi\right)$.

Here, $v=0.3$ and all solutions are again given with respect to the reference configuration.

This benchmark was simulated with standard MPM and PS-MPM, using an unstructured triangular grid with material points initialised uniformly over the domain, as shown in Fig. 12. A time step size of $\Delta t=2.25 \cdot 10^{-4} \mathrm{~s}$ was chosen, which results in a Courant number of $C \approx 0.72$. For the adopted parameters, the imposed solution in Eqs. 40-41 has period $T=0.02 \mathrm{~s}$.

\subsubsection{Grid-crossing error}

First, it is shown that the grid-crossing error typical for standard MPM does not occur in PS-MPM, by comparing the normal horizontal stress resulting from these methods (see Fig. 13). The configurations for PS-MPM and standard MPM contain the same number of particles (5120, 4 times as many as in Fig. 12) and a comparable number of basis functions, 289 for standard MPM and 243 for PS-MPM. As expected, the stress field in standard MPM suffers severely from grid-crossing, whereas with PS-MPM a smooth stress field is observed. The same conclusion can also be drawn when investigating individual particle stresses over time, as is shown in Fig. 14. For this figure, the particle positioned at $\left(x^{0}, y^{0}\right) \approx(0.25 \mathrm{~m}, 0.47 \mathrm{~m})$ has been traced throughout the simulation. The figure depicts the stress and trajectory of the particle. For standard MPM, it is observed that the stress profile is highly oscillatory, resulting in a disturbed trajectory. PS-MPM shows no oscillations, but only a small offset, which was found to disappear upon further refinement of the grid.

\subsubsection{Spatial convergence}

PS-MPM with quadratic basis functions is expected to show third-order spatial convergence. To determine the spatial convergence, the time averaged root-mean-squared (RMS) error is used:

RMS-error $=\sqrt{\frac{\sum_{i=1}^{n_{t}} \sum_{p=1}^{n_{p}}\left|x_{p}\left(t_{i}\right)-\hat{x}_{p}\left(t_{i}\right)\right|^{2}}{n_{p} n_{t}}}$,

where $n_{t}$ denotes the total number of time steps of the simulation. Under the assumption that the spatial error is much larger than the error produced by the numerical integration and time-stepping scheme, the spatial convergence of standard MPM and PS-MPM is determined by varying the typical element length $h$. This length was defined as the average edge length for standard MPM and the average sub-triangle edge length for PS-MPM. It has been observed that the timeintegration error is indeed sufficiently small, but the number of particles required for an adequately accurate integration increases rapidly as $h$ decreases.

Figure 15 shows the spatial convergence of both standard MPM and PS-MPM for different numbers of particles per element on an unstructured grid. Provided that a sufficient number of particles is adopted, standard MPM shows secondorder spatial convergence, and PS-MPM converges with third order. Both orders of convergence correspond to the order of the projection-error, as shown in Sect. 3.5. Besides higherorder spatial convergence, PS-MPM also results in a smaller RMS-error compared to standard MPM, even for the coarsest grid.

To achieve the optimal convergence order for both standard MPM and PS-MPM, the number of integration particles required increases rapidly, as the integration error otherwise dominates the total error, as shown in Fig. 15. Note that the convergence rate in the number of particles for both standard MPM and PS-MPM is measured to be of first order. However, for a fixed typical element length $h$ and number of particles per element, the use of PS-MPM leads to a lower RMS-error, illustrating the higher accuracy per degree of freedom. The inaccurate integration in MPM due to the use of particles as integration points is known to limit the spatial convergence. Different measures have been proposed to decrease the quadrature error in MPM based on function reconstruction techniques like MLS [13], cubic splines [37] and Taylor least squares [39]. The combination of PS-MPM with function reconstruction techniques to obtain optimal convergence rates with a moderate number of particles is subject to future research to further improve PS-MPM for practical applications.

\subsection{Column under self-weight}

In the previous examples, we considered PS-MPM with a consistent mass matrix. However, lumping of the mass matrix is common practice in many applications of MPM, as it speeds up the simulation and increases the numerical stability. In case elements become (almost) empty, the consistent mass matrix in Eq. 13 has very small entries and becomes ill-conditioned, leading to stability issues, which is a wellknown phenomena in MPM [32]. Using a lumped version of the mass matrix to solve for the acceleration and velocity fields is known to overcome the ill-conditioning [32]. However, as explained in Sect. 3.5, lumping within PS-splines can cause spatial oscillations. We show that this is indeed the case and propose an alternative based to lumping to overcome the ill-conditioning of the mass matrix while mitigating spatial oscillations as much as possible.

First, we demonstrate that the standard lumping technique is poorly suited for PS-MPM, by considering a solid col- 
Fig. 12 The exact solution in which particles (marked with dots) move back and forth along the marked vectors (left) and the unstructured grid with the initial particle configuration (right)
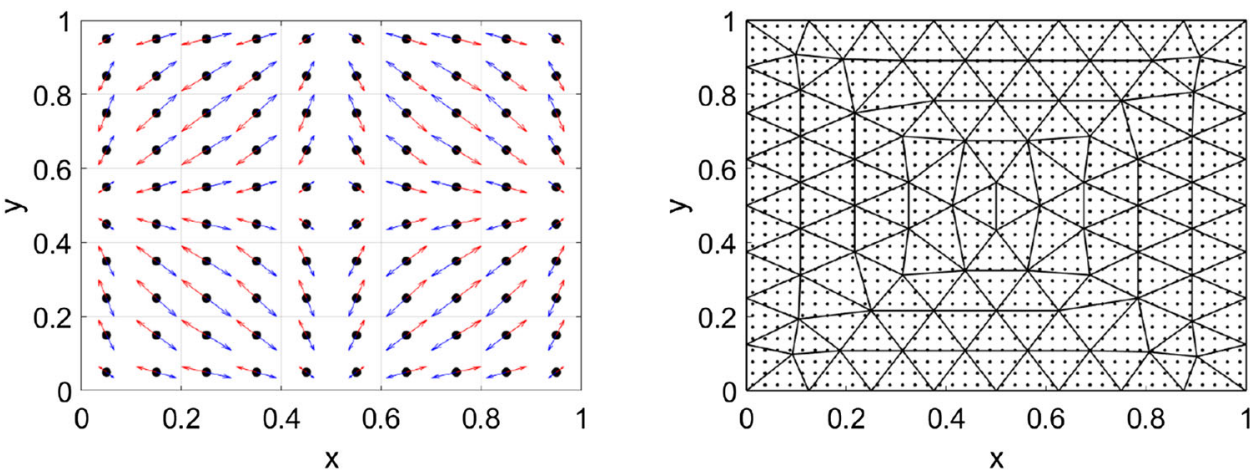

Fig. 13 The interpolated particle stress in the $x$-direction at $t=0.016 \mathrm{~s}$

Fig. 14 The stress (left) and movement (right) of a single particle over time (in seconds) for both standard MPM and PS-MPM compared to the exact solution

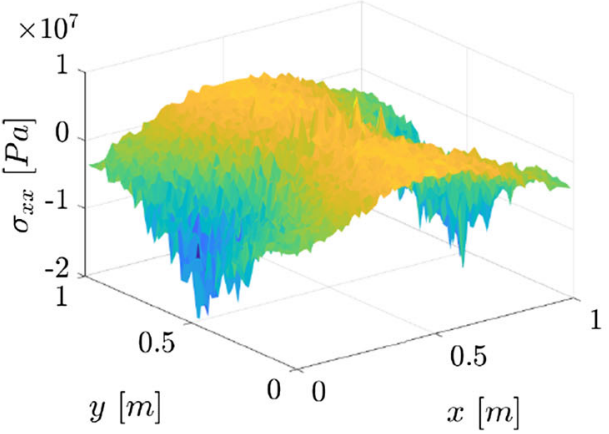

Standard MPM

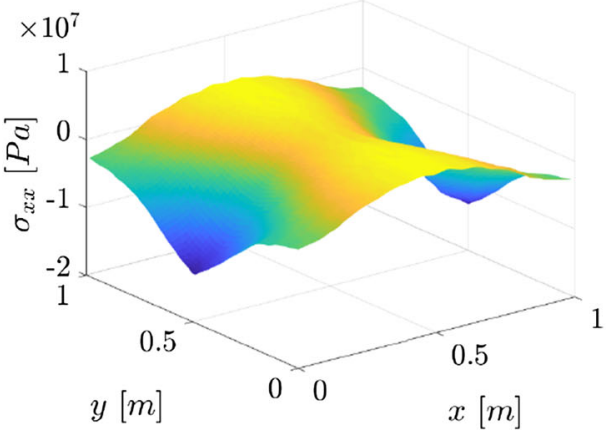

PS-MPM

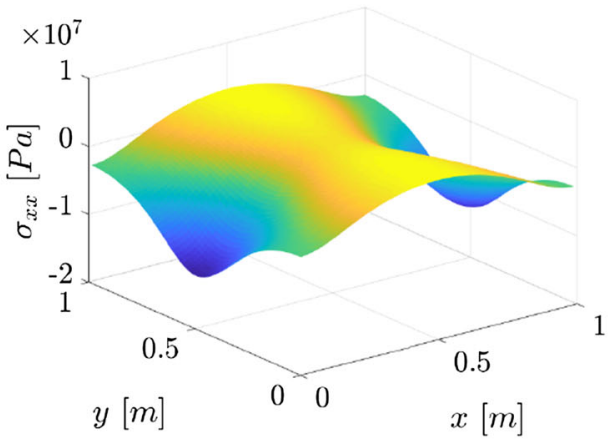

Exact
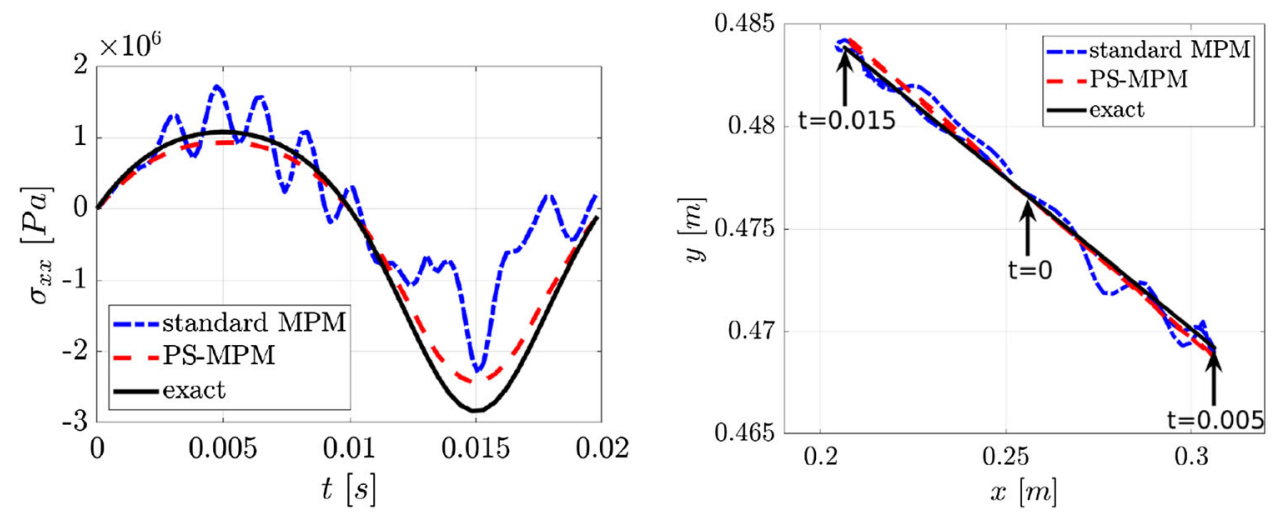
Fig. 15 Spatial convergence (top) and convergence in the number of particles per element (ppe) (bottom) for both standard MPM (left) and PS-MPM (right)
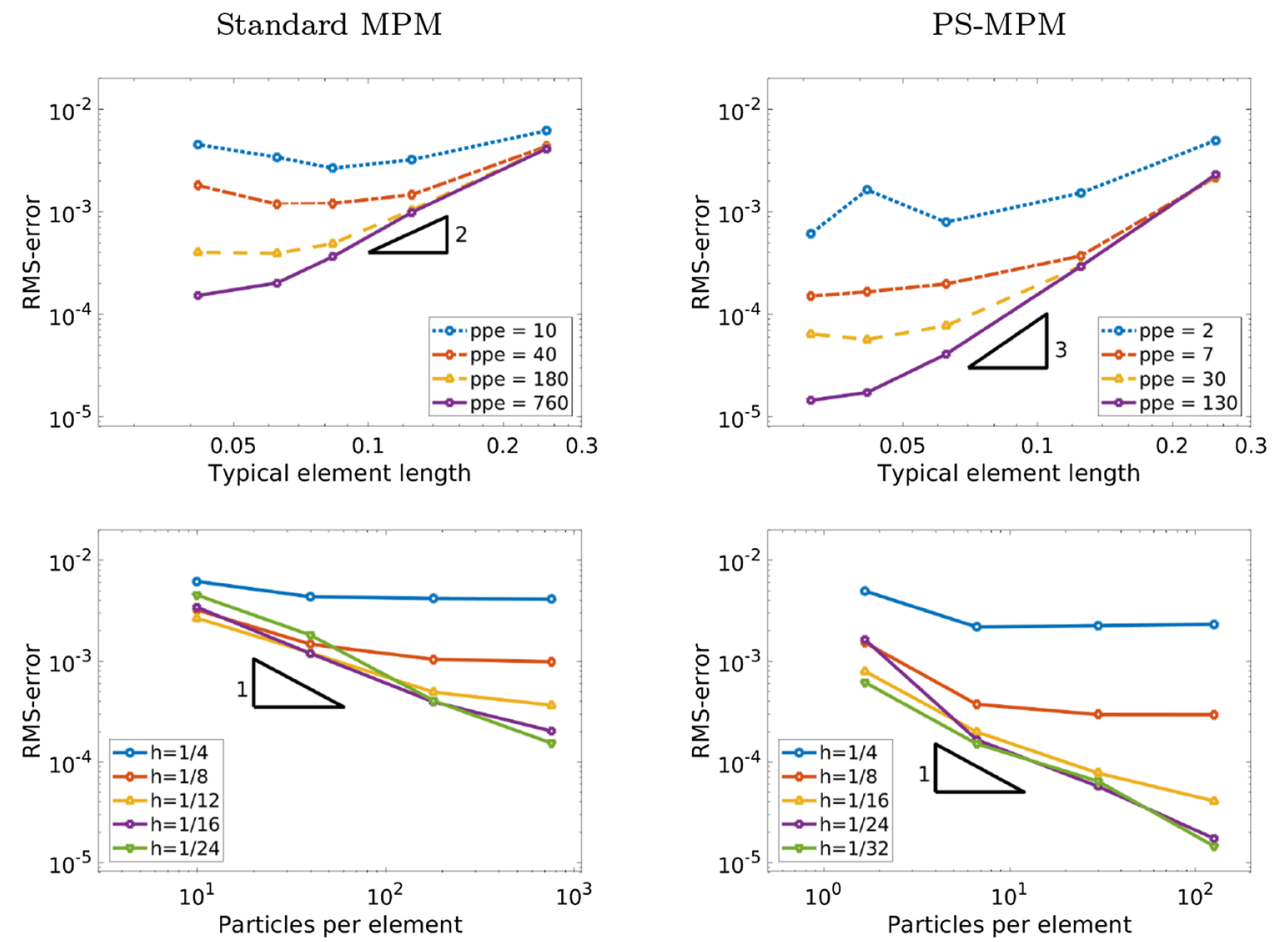

umn under self-weight, as shown in Fig. 16. The column in this exemplary benchmark is modelled as a linear-elastic material. It is fixed in all directions at the bottom, and completely free at the top. At the left and right boundary, we impose a free-slip boundary condition for movement in the $y$-direction, but the displacement in the $x$-direction is fixed to be zero. The column is compressed by gravity due to its self-weight.

The solid column is modelled with density $\rho=1$. $10^{3} \mathrm{~kg} / \mathrm{m}^{3}$, Young's modulus $E=1 \cdot 10^{5} \mathrm{~Pa}$, Poisson ratio $v=0$, gravitational acceleration $g=-9.81 \mathrm{~m} / \mathrm{s}^{2}$, height $H=1 \mathrm{~m}$ and width $W=0.1 \mathrm{~m}$. The maximum strain when adopting these parameters is approximately $18 \%$. Figure 16 illustrates the grid considered for this benchmark as well as the particle positions at maximal deformation.

Due to the fact that part of the grid becomes empty, the use of a consistent mass matrix leads to stability issues with unlumped PS-MPM. The use of a lumped mass matrix within PS-MPM restores the stability, but causes spatial oscillations in the stress profile, as was also discussed in Sect. 3.5. The oscillations degrade the solution of the displacement and velocity as well, as shown in Fig. 17 (left column). Since these spatial oscillations are typical for lumped PS-MPM, common remedies to solve problems due to lumping the mass matrix, like the momentum formulation method [33] or distribution coefficient method [17], do not reduce the spatial oscillations in lumped PS-MPM. Instead, we propose the use of partial lumping.
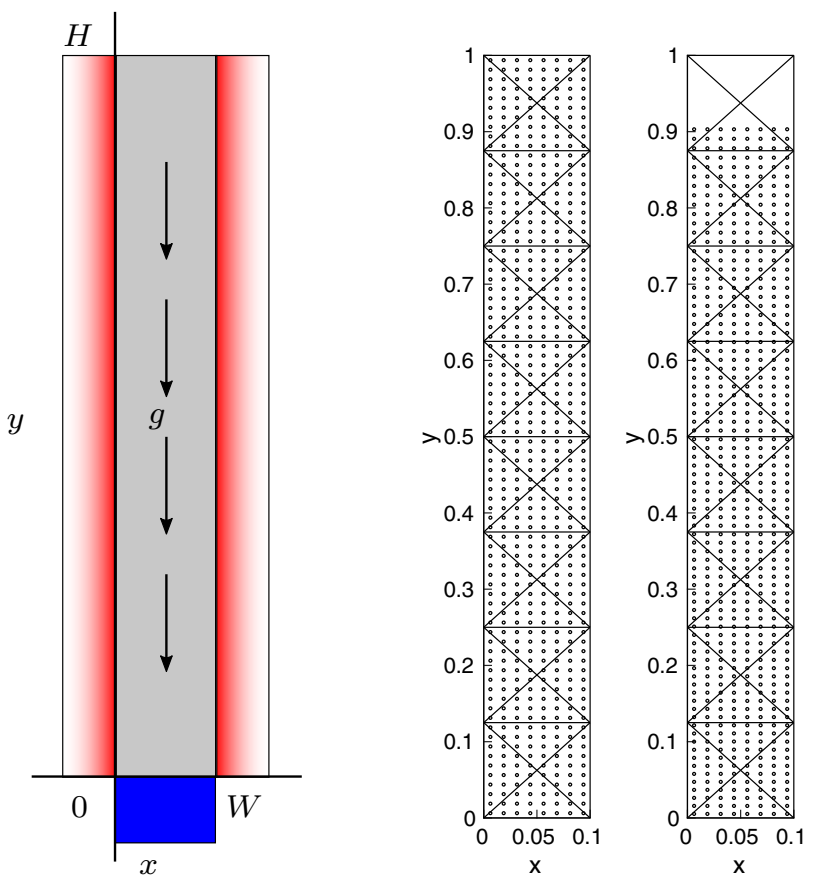

Fig. 16 The column and a discretisation with the expected material point positions at $t=0$ and $t=0.186$

\subsubsection{Partial lumping to mitigate spatial oscillations}

As full lumping within PS-MPM causes spatial oscillations, we will instead consider a partial-lumping approach to overcome the stability issues due to almost empty elements, while 
Fig. 17 A comparison of fully lumped PS-MPM (left) and partially lumped PS-MPM (right) for simulation of a solid column up to $t=2.5 \mathrm{~s}$. For the energy graphs, $E_{k i n}$ denotes kinetic energy, $E_{p o t}$ the potential energy due to strain, $E_{\text {grav }}$ the gravitational energy and $E_{t o t}$ the total energy

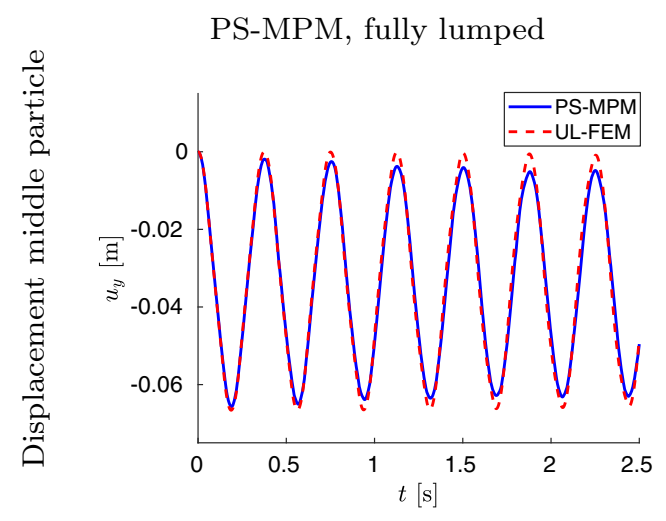

PS-MPM, partially lumped
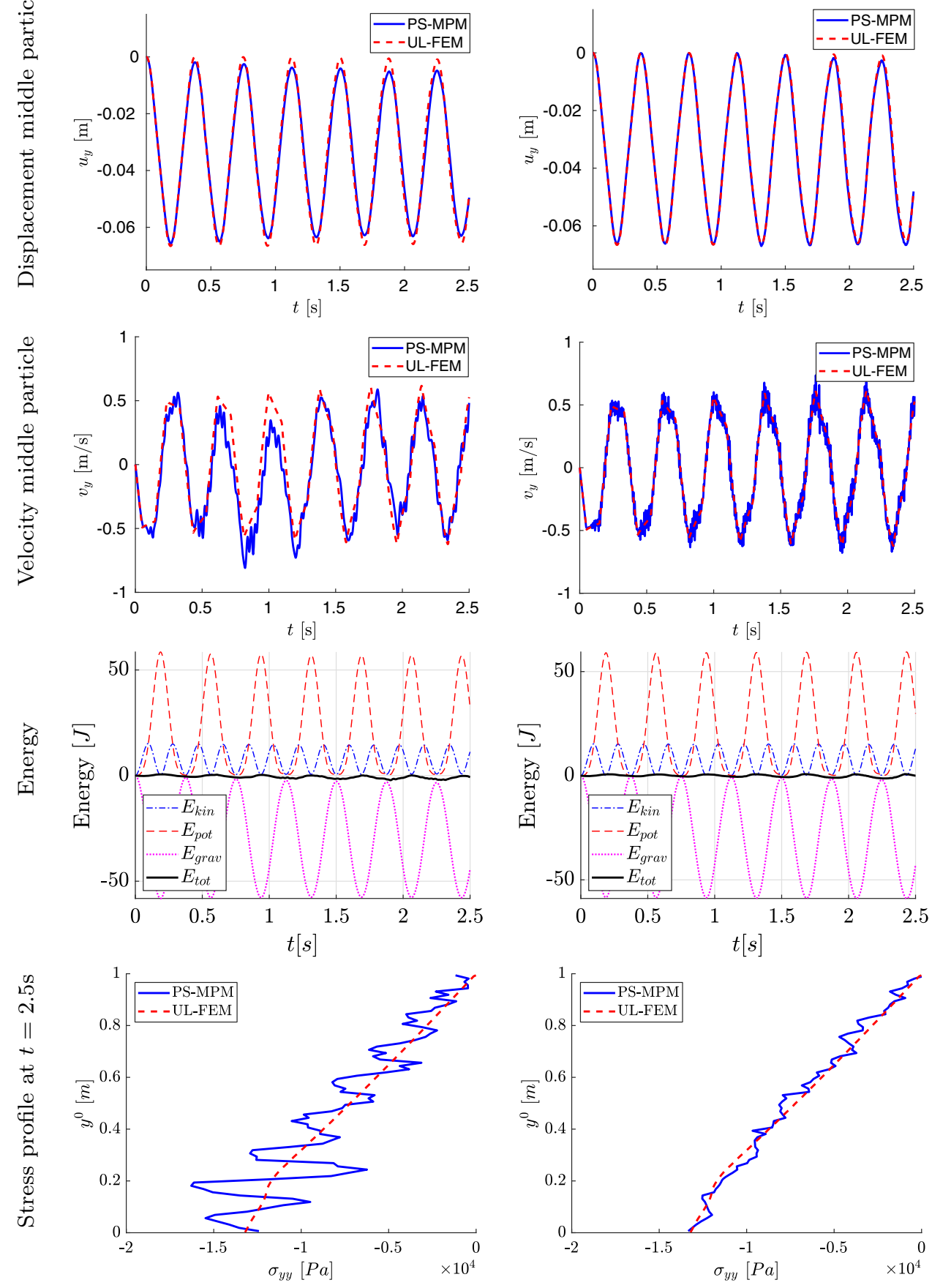

at the same time minimising spatial oscillations due to lumping. Partial lumping only lumps those rows responsible for the ill-conditioning. These rows typically correspond to the basis functions in the part of the grid where very few particles are left. By only lumping these few rows, the simulation is stabilised, while introducing a much smaller lumping error compared to full lumping. Note that the choice of rows to lump is crucial to ensure stability. Lumping more rows increases the robustness of the simulation, but decreases the accuracy. The trade-off between stability and accuracy within partially lumped PS-MPM should be reconsidered for each benchmark.

For the column under self-weight, we implemented partial lumping by lumping all rows corresponding to a basis function with at least one empty main-triangle in its molecule. Additional empty elements are added at the top of the column, to ensure that the top-most basis functions are always lumped. Figure 17 (right column) shows the displacement 
Fig. 18 The equilibrium stress at individual particles in a damped solid column. The numerical solutions were determined with fully lumped and partially lumped PS-MPM at $t=10 \mathrm{~s}$, at which the solution was in equilibrium

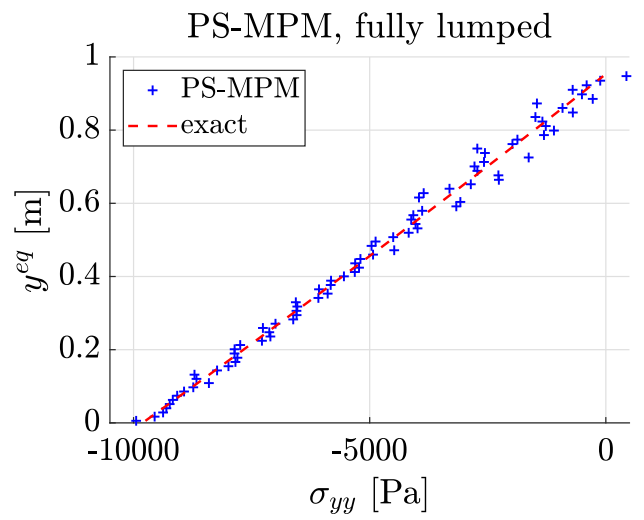

and velocity of a particle over time, as well as the stress profile at $t=2.5 \mathrm{~s}$ obtained with partially lumped PS-MPM. Compared to the results obtained with fully lumped PSMPM, shown in Fig. 17 (left), the stress profile, the velocity and displacement over time significantly improve. Furthermore, the total energy fluctuates less for partially lumped PS-MPM than for fully lumped PS-MPM and also shows less numerical dissipation. However, for very long simulations, partially lumped PS-MPM requires smaller time steps than fully lumped PS-MPM in order to remain stable.

Finally, we compared the equilibrium solution for the stress profile in the solid column with partial and full lumping. The analytical equilibrium solution is given by [41]:

$\sigma_{y y}\left(y^{e q}\right)=\sigma_{y y}(0) \frac{\left(1-y^{e q} / H\right)+\kappa\left(y^{e q} / H\right)}{1-\kappa(1-\kappa)\left(y^{e q} / H\right)}$,

where $y^{e q}$ refers to the equilibrium position of the particles. Furthermore, $\sigma_{y y}(0)=H \rho g$ and $\kappa=\frac{\sigma_{y y}(0)}{2 E}$. To obtain the static solution based on the dynamic calculations, a damping term has been added:

$\boldsymbol{F}_{k}^{\mathrm{damp}}=-\alpha \operatorname{sign}\left(\hat{\boldsymbol{v}}_{k}\right)\left(\boldsymbol{F}_{k}^{\mathrm{trac}}-\boldsymbol{F}_{k}^{\mathrm{int}}+\boldsymbol{F}_{k}^{\mathrm{body}}\right)$

where, $\alpha=0.6$. The damping force damps the PS-MPM solution at each time step in the opposite direction of the velocity field. The equilibrium solution has then been determined by simulating the system with both fully lumped and partially lumped PS-MPM until a stable solution was reached. Both the analytical and numerical static solutions are shown in Fig. 18. For fully lumped PS-MPM, the spatial oscillations in the stress profile remain visible in the equilibrium solution. However, the stress profile of partially lumped PS-MPM is in close agreement with the exact solution.

Hence, the use of partial lumping within PS-MPM combines the advantages of adopting a consistent and lumped mass matrix, while minimising their drawbacks, and thereby improves the overall performance of the method. Future research will focus on techniques to further mitigate or fully overcome the oscillations caused by lumping, in particular when PS-MPM is applied for practical problems.

\section{Conclusion}

In this paper, we presented an alternative for B-spline MPM suited for unstructured triangulations using piecewise quadratic $C^{1}$-continuous Powell-Sabin spline basis functions. The method combines the benefits of smooth, higher-order basis functions with the geometric flexibility of an unstructured triangular grid. PS-MPM yields a mathematically sound approach to eliminate grid-crossing errors, due to the smooth gradients of the basis functions. As a first validation, a vibrating bar was considered, for which the PSMPM solution yields accurate results on a relatively coarse grid. Numerical simulations obtained for a unit square undergoing axis-aligned displacement have shown higher-order convergence for the particle stresses and displacements.

The use of a lumped mass matrix to increase stability within PS-MPM leads to spatial oscillations in the stress profile. A partial lumping strategy was proposed to combine the advantages of adopting a consistent and lumped mass matrix, which successfully mitigates this issue. Investigation of alternative unstructured spline technologies, in particular, refinable $C^{1}$ splines on irregular quadrilateral grids [21] is underway.

\section{Compliance with ethical standards}

Conflict of interest Pascal de Koster has received funding support from Dutch research institute Deltares.

Open Access This article is licensed under a Creative Commons Attribution 4.0 International License, which permits use, sharing, adaptation, distribution and reproduction in any medium or format, as long as you give appropriate credit to the original author(s) and the source, provide a link to the Creative Commons licence, and indicate if changes were made. The images or other third party material in this article are included in the article's Creative Commons licence, unless indicated otherwise in a credit line to the material. If material 
is not included in the article's Creative Commons licence and your intended use is not permitted by statutory regulation or exceeds the permitted use, you will need to obtain permission directly from the copyright holder. To view a copy of this licence, visit http://creativecomm ons.org/licenses/by/4.0/.

\section{References}

1. Andersen S, Andersen L (2010) Modelling of landslides with the material-point method. Comput Geosci 14:137-147

2. Bardenhagen S, Kober E (2004) The generalized interpolation material point method. Comput Model Eng Sci 5(6):477-496

3. Bazilevs Y, Moutsanidis G, Bueno J, Kamran K, Kamensky D, Hillman MC, Gomez H, Chen JS (2017) A new formulation for airblast fluid-structure interaction using an immersed approach: part II-coupling of IgA and meshfree discretizations. Comput Mech 60(1):101-116

4. Courant R, Friedrichs K, Lewy H (1928) Über die partiellen Differenzengleichungen der mathematischen Physik. Math Ann 100(1):32-74

5. Cromer A (1981) Stable solutions using the euler approximation. Am J Phys 49(5):455-459

6. De Koster P (2018) Towards a material point method with PowellSabin spline basis functions. Master's thesis, Delft University of Technology

7. Dhakal TR, Zhang DZ (2016) Material point methods applied to one-dimensional shock waves and dual domain material point method with sub-points. J Comput Phys 325:301-313

8. Dierckx P (1997) On calculating normalized Powell-Sabin Bsplines. Comput Aided Geom Des 15(1):61-78

9. Dierckx P, Van Leemput S, Vermeire T (1992) Algorithms for surface fitting using Powell-Sabin splines. IMA J Numer Anal 12(2):271-299

10. Donea J, Huerta A, Ponthot J, Rodriguez-Ferran A (2004) Arbitrary Lagrangian-Eulerian methods. In: Stein R, de Borst R, Hughes T (eds) Encyclopedia of Computational Mechanics. Wiley, New York

11. Gan Y, Sun Z, Chen Z, Zhang XYL (2017) Enhancement of the material point method using B-spline basis functions. Int J Numer Methods Eng 113:411-431

12. Gaume J, Gast T, Teran J, van Herwijnen A, Jiang C (2018) Dynamic anticrack propagation in snow. Nat Commun 9(1):3047

13. Gong M (2015) Improving the material point method. Ph.D. thesis, The University of New Mexico

14. Guo Y, Nairn J (2017) Simulation of dynamic 3D crack propagation within the material point method. Comput Model Eng Sci 113(4):389-410

15. Hughes TJ, Evans JA, Reali A (2014) Finite element and NURBS approximations of eigenvalue, boundary-value, and initial-value problems. Comput Methods Appl Mech Eng 272:290-320

16. Leavy R, Guilkey J, Phung B, Spear A, Brannon R (2019) A convected-particle tetrahedron interpolation technique in the material-point method for the mesoscale modeling of ceramics. Comput Mech 64(3):563-583

17. Ma X, Giguere P, Jayaraman B, Zhang D (2010) Distribution coefficient algorithm for small mass nodes in material point method. $\mathrm{J}$ Comput Phys 229:7819-7833

18. Manni C, Sablonniere P (2007) Quadratic spline quasi-interpolants on Powell-Sabin partitions. Adv Comput Math 26(1-3):283-304

19. Motlagh YG, Coombs WM (2017) An implicit high-order material point method. Proc Eng 175:8-13

20. Nairn JA (2003) Material point method calculations with explicit cracks. Comput Model Eng Sci 4(6):649-664

21. Nguyen T, Peters J (2016) Refinable $C^{1}$ spline elements for irregular quad layout. Comput Aided Geom Des 43:123-130
22. Nguyen VP, Nguyen CT, Rabczuk T, Natarajan S (2017) On a family of convected particle domain interpolations in the material point method. Finit Elem Anal Des 126:50-64

23. Powell MJ, Sabin MA (1977) Piecewise quadratic approximations on triangles. ACM Trans Math Softw (TOMS) 3(4):316-325

24. Sablonnière $P$ (1987) Error bounds for Hermite interpolation by quadratic splines on an $\alpha$-triangulation. IMA J Numer Anal 7(4):495-508

25. Sadeghirad A, Brannon RM, Burghardt J (2011) A convected particle domain interpolation technique to extend applicability of the material point method for problems involving massive deformations. Int J Numer Meth Eng 86(12):1435-1456

26. Sadeghirad A, Brannon RM, Guilkey J (2013) Second-order convected particle domain interpolation (CPDI2) with enrichment for weak discontinuities at material interfaces. Int J Numer Meth Eng 95(11):928-952

27. Speleers H, Manni C, Pelosi F, Sampoli ML (2012) Isogeometric analysis with Powell-Sabin splines for advection-diffusionreaction problems. Comput Methods Appl Mech Eng 221:132-148

28. Steffen M, Kirby RM, Berzins M (2008) Analysis and reduction of quadrature errors in the material point method (MPM). Int J Numer Meth Eng 76(6):922-948

29. Steffen M, Kirby RM, Berzins M (2010) Decoupling and balancing of space and time errors in the material point method (MPM). Int J Numer Meth Eng 82(10):1207-1243

30. Steffen M, Wallstedt P, Guilkey J, Kirby R, Berzins M (2008) Examination and analysis of implementation choices within the material point method (MPM). Comput Model Eng Sci 32(2):107127

31. Stomakhin A, Schroeder C, Chai L, Teran J, Selle A (2013) A material point method for snow simulation. ACM Trans Gr (TOG) 32(4): 102

32. Sulsky D, Chen Z, Schreyer HL (1994) A particle method for history-dependent materials. Comput Methods Appl Mech Eng 118(1-2):179-196

33. Sulsky D, Kaul A (2004) Implicit dynamics in the material-poin method. Comput Methods Appl Mech Eng 193:1137-1170

34. Sulsky D, Schreyer H, Peterson K, Kwok R, Coon M (2007) Using the material point method to model sea ice dynamics. J Geophys Res 76:922-948

35. Sulsky D, Schreyer L (2004) MPM simulation of dynamic material failure with a decohesion constitutive model. Eur J Mech A/Solids 23(3):423-445

36. Ten Thije R, Akkerman R, Huétink J (2007) Large deformation simulation of anisotropic material using an updated Lagrangian finite element method. Comput Methods Appl Mech Eng 196(3334):3141-3150

37. Tielen R, Wobbes E, Möller M, Beuth L (2017) A high order material point method. Proc Eng 175:265-272

38. Wallstedt P, Guilkey J (2008) An evaluation of explicit time integration schemes for use with the generalized interpolation material point method. J Comput Phys 227(22):9628-9642

39. Wobbes E, Möller M, Galavi V, Vuik C (2019) Conservative Taylor least squares reconstruction with application to material point methods. Int J Numer Meth Eng 117(3):271-290

40. Zabala F, Alonso E (2011) Progressive failure of Aznalcóllar dam using the material point method. Géotechnique 61(9):795-808

41. Zhang DZ, Ma X, Giguere PT (2011) Material point method enhanced by modified gradient of shape function. J Comput Phys 230(16):6379-6398

Publisher's Note Springer Nature remains neutral with regard to jurisdictional claims in published maps and institutional affiliations. 